\title{
Classification of Electric Vehicle Fleets Considering the Complexity of Fleet Charging Schedules
}

\author{
Nicki Bodenschatz, Diana Schramm, Markus Eider, Andreas Berl \\ Deggendorf Institute of Technology \\ [nicki.bodenschatz, diana.schramm, markus.eider, andreas.berl]@th-deg.de
}

\begin{abstract}
The increasing number of electric vehicles has a growing impact on the power grid. Therefore, charging processes need to be scheduled. In order to clarify and solve the challenges of scheduling the charging of a fleet, this paper analyses, categorizes and classifies different kinds of fleets of electric vehicles.

CCS Concepts• Theory of computation $\rightarrow$ Scheduling algorithms - Theory of computation $\rightarrow$ Discrete optimization - Theory of computation $\rightarrow$ Packing and covering problems $\bullet$ Theory of computation $\rightarrow$ Algorithm design techniques
\end{abstract}

\section{KEYWORDS}

Electric vehicles, vehicle fleet, fleet classification, charging scheduling, NP-completeness

\section{ACM Reference format:}

Nicki Bodenschatz, Diana Schramm, Markus Eider and Andreas Berl.2018 Classification of Electric Vehicle Fleets Considering the Complexity of Fleet Charging Schedules. ACM e-Energy conference, Karlsruhe, Germany, June 2018, 2 pages. https://doi.org/10.1145/3208903.3212056

\section{INTRODUCTION}

The number of Electric Vehicles (EVs) is continuously growing, especially in the business sector. This steady growing demand of energy to charge the fleet could harm the power grid, especially if the fleet charging is not coordinated with regards to the available energy capacity of the grid. To prevent this, the charging processes have to be scheduled. To solve this Charging Scheduling Problem (CSP), adequate algorithms and heuristics have to be used.

In the CSP the goal is to find a cost optimized charging plan for the whole fleet, which is valid under certain restrictions, like the available grid capacity. It refers to the NP-complete problems which have tremendous computation time for large problem instances. However, there are existing algorithms and heuristics, which are capable of solving the CSP for EV fleets.

In the related work there are different approaches to solve the CSP. However, there are almost no hints, which algorithm or heuristic is

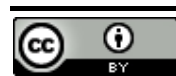

This work is licensed under a Creative Commons Attribution International 4.0 License.

e-Energy '18, June 12-15, 2018, Karlsruhe, Germany

(C) 2018 Copyright held by the owner/author(s).

ACM ISBN 978-1-4503-5767-8/18/06.

https://doi.org/10.1145/3208903.3212056 adequate for a specific fleet type. To answer this, it is necessary to understand the term fleet in the context of the CSP.

The goal of this paper is to establish a class system for fleets in the CSP to make them comparable and distinguishable from each other. These classes uncouple the understanding of the fleet business from the modelling of the CSP. They can be used as a basis to find the right heuristic or algorithm for a certain fleet.

To do this there is a discussion in the second Section about the solving methods in the related work. In the third Section, the initial situation of the CSP is explained and the constraints are defined. On this fundament, the single components are analyzed in the fourth Section. This analyzation allows a derivation of the suggested classes. In the fifth Section, there is a short conclusion of the opportunities of these classes.

\section{RELATED WORK}

This Section provides an overview of different heuristics and algorithms to solve the CSP. Xi and Siohansi [8] propose a decentralized price/quantity based approach. Mixed Integer Programming solution (MIP) is applied as described by Álvaro and Fraile-Ardanuy [1], Detzler and Karnouskos [2] or Sassi and Oulamara [6]. The latter also use a Min-Cost-Flow-Problem based heuristic [6]. Other approaches include for example a binary linear programming method [5] or stochastic approaches [4]. Also a partition into time slots allows discrete solving approaches [3-7]. All authors present different methods to solve the CSP, but they often use different constraints of the CSP and it is unclear which method applies to which fleet type. This paper tackles this problem.

\section{CHARGING SCHEDULING PROBLEM}

This Section explains the CSP and its constraints.

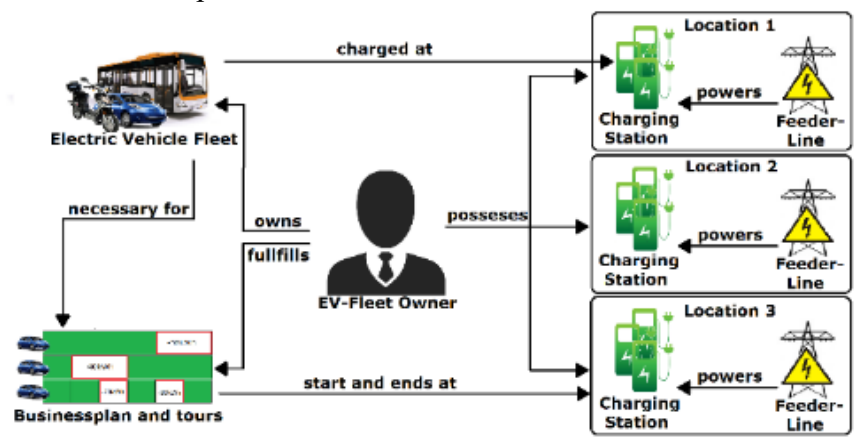

Figure 1 - Initial situation of the CSP 
ACM e-Energy 2018, Karlsruhe, Germany

\begin{tabular}{l|c|c|c|c|c|c|c|c|c|c|c|c|c|c|c|c}
\hline Scenario & 1 & 2 & 3 & 4 & 5 & 6 & 7 & 8 & 9 & 10 & 11 & 12 & 13 & 14 & 15 & 16 \\
\hline Knowledge of tour start time & $\mathrm{Y}$ & $\mathrm{Y}$ & $\mathrm{Y}$ & $\mathrm{Y}$ & $\mathrm{Y}$ & $\mathrm{Y}$ & $\mathrm{Y}$ & $\mathrm{Y}$ & $\mathrm{N}$ & $\mathrm{N}$ & $\mathrm{N}$ & $\mathrm{N}$ & $\mathrm{N}$ & $\mathrm{N}$ & $\mathrm{N}$ & $\mathrm{N}$ \\
\hline Knowledge of tour end time & $\mathrm{Y}$ & $\mathrm{Y}$ & $\mathrm{Y}$ & $\mathrm{Y}$ & $\mathrm{N}$ & $\mathrm{N}$ & $\mathrm{N}$ & $\mathrm{N}$ & $\mathrm{Y}$ & $\mathrm{Y}$ & $\mathrm{Y}$ & $\mathrm{Y}$ & $\mathrm{N}$ & $\mathrm{N}$ & $\mathrm{N}$ & $\mathrm{N}$ \\
\hline Knowledge of energy consumption & $\mathrm{Y}$ & $\mathrm{Y}$ & $\mathrm{N}$ & $\mathrm{N}$ & $\mathrm{Y}$ & $\mathrm{N}$ & $\mathrm{N}$ & $\mathrm{Y}$ & $\mathrm{Y}$ & $\mathrm{N}$ & $\mathrm{N}$ & $\mathrm{Y}$ & $\mathrm{Y}$ & $\mathrm{N}$ & $\mathrm{N}$ & $\mathrm{Y}$ \\
\hline fixed tours & $\mathrm{Y}$ & $\mathrm{N}$ & $\mathrm{Y}$ & $\mathrm{N}$ & $\mathrm{Y}$ & $\mathrm{Y}$ & $\mathrm{N}$ & $\mathrm{N}$ & $\mathrm{Y}$ & $\mathrm{Y}$ & $\mathrm{N}$ & $\mathrm{N}$ & $\mathrm{Y}$ & $\mathrm{Y}$ & $\mathrm{N}$ & $\mathrm{N}$ \\
\hline Class & \multicolumn{3}{|c|}{$\begin{array}{c}\text { Known departure } \\
\text { fleets }\end{array}$} & \multicolumn{3}{c|}{$\begin{array}{c}\text { Known arrival } \\
\text { fleets }\end{array}$} & $\begin{array}{c}\text { Arbitrary presence } \\
\text { fleets }\end{array}$ \\
\hline
\end{tabular}

Table 1 - Fleet condition combinations and classes

The EV-Fleet Owner (EFO) has different types of EVs, which are necessary to fulfill the tours of the business plan. These tours start and end at locations of the EFO. There, the EVs have to be charged at Charging Stations (CSs). The CSs are supplied with energy by one feeder line (on joint grid capacity) per location (see Figure 1). The goal is to schedule the charging processes in a cost optimal way, so that the business plan can still be fulfilled. For these charging processes, there are constraints. (1) Charging availability: EVs can only charge when they are not on a tour. (2) CS-EV-Compatibility: The CS adapter and the EV adapter must fit together. (3), Location matching: An EV can only use a CS at the same place. (4) Charging powers of CS/EV: The minimal and maximal charging power of a $\mathrm{CS} / \mathrm{EV}$ adapter have to be respected. (5) Grid capacity compliance: The drain of charged energy must not exceed the grid capacity. (6) Single charging of EV at CS adapter: Only one EV can charge at a single CS adapter at the same time. (7) No multiple charging processes by one EV: One EV can only charge at one CS at the same time (8) Battery capacity compliance: The State of Charge (SoC) of an EV cannot be below $0 \%$ or above $100 \%$. (9) Tour readiness: Every tour must be carried out by one EV. The SoC must be higher than the energy consumption of the tour.

\section{CLASSIFICATION APPROACH}

The constraints of the CSP are the same for every kind of fleet, but there is a difference in the specific characteristic of the CSP for every fleet. In order to reduce this large variety, fleet classes are established to cluster and confine the CSP complexity. This requires a closer look on the input information of the CSP.

\subsection{Input information analysis}

\begin{tabular}{lll}
\hline Input information & Constraint & Result \\
\hline EV Charging adapter & 2,9 & Technical input \\
EV Charging power & $4,5,9$ & Technical input \\
EV Battery capacity & 8,9 & Technical input \\
CS Charging adapters & 2,9 & Technical input \\
CS Charging Power & 4,9 & Technical input \\
CS location & 3,9 & Technical input \\
EV location & 3,9 & Technical input \\
Grid capacity & 5,9 & Technical input \\
\hline Grid energy price & - & Optimization criteria \\
\hline EV to tour assignment & $1,3,9$ & Estimation necessary \\
Tour start & 1,9 & Estimation necessary \\
Tour end & 1,9 & Estimation necessary \\
deltaSoC & 9 & Estimation necessary \\
\hline
\end{tabular}

Table 2 - Overview input information
In the first column of Table 2, is the type of input information for the EVs, the CSs, the grid and the tours. In the second column are the constraints, which are affected by the information in the first column. In the third, there is an interpretation of the information. Every technical information is necessary to create a valid charging schedule. For example, it is necessary to know the EV charging adapter, to charge at a compatible CS. The grid energy price is necessary to optimize the charging costs. The last four rows, are the most interesting ones, because some EFOs cannot provide this information, due to their business model. For example, a taxi fleet cannot plan the tours in advance. They have to react to the demands of customers. In consequence, it is not clear, when these EVs are available for charging, which makes a scheduling impossible. To make an optimization possible, it is necessary to establish algorithms which calculate these parameters in advance. These algorithms make the CSP much more complex.

\subsection{Fleet classification approach}

If the last for rows of input parameters are missing, algorithms are necessary to estimate them. This increases the complexity. Based on this, we suggest to classify the fleets by this input information. Therefore, the fleets are classified in the following four classes: 1. known arrival and departure fleets, 2. known departure fleets, 3. known arrival fleets and 4. arbitrary presence fleets.

Based on the combination of these conditions and the resulting 16 different fleet scenarios we identified four classes of fleets (see Table 1). In this table, all combinations of the four conditions are illustrated. Every condition can be formulated as a question that can be answered with yes $(\mathrm{Y})$ or no $(\mathrm{N})$.

\section{CONCLUSION}

Overall this paper provides a general information of the input parameters and the constraints of the CSP. It also suggests different classes for the fleets. By dividing the fleets in these classes, it is possible, to solely focus on the modelling and the solving of the CSP. It is not necessary to understand the business goals or the fleet type. It does not matter if it is a taxi fleet, a bus fleet or a train fleet. If the classes and the technical inputs can be derived from it, a CSP can be established. This uncoupling of the CSP modelling from the business interpretation of a fleet, makes the charging cost optimization more straightforward and much easier. It makes it also possible to arrange the fleet in specific classes. This allows it to derive and compare the mathematical model, and therefore also the complexity of the different EV fleets. 


\section{ACKNOWLEDGEMENTS}

This research was funded by the European Union Horizon 2020 research and innovation programme under grant $\mathrm{N}^{\circ} 713864$.

\section{REFERENCES}

[1] Alvaro, R. and Fraile-Ardanuy, J. 2015. Charge scheduling strategies for managing an electric vehicle fleet parking. IEEE EUROCON $2015-$ International Conference on Computer as a Tool (EUROCON) (Sep. 2015), 1-6.

[2] Detzler, S. and Karnouskos, S. 2015. A model and an evolutionary algorithmic approach towards optimization of Electric Vehicle fleet charging. 2015 International Symposium on Smart Electric Distribution Systems and Technologies (EDST) (Sep. 2015), 20-25.

[3] Yuchang Wang and Thompson, J. 2017. Admission and scheduling mechanism for electric vehicle charging with renewable energy. 2017 IEEE International Conference on Communications Workshops (ICC Workshops) (May 2017), 1304-1309.

[4] Korkas, C.D. et al. 2017. A cognitive stochastic approximation approach to optimal charging schedule in electric vehicle stations. 201725 th Mediterranean Conference on Control and Automation (MED) (Jul. 2017), 484-489.

[5] Nguyen, V.-L. et al. 2014. Charging strategies to minimize the peak load for an electric vehicle fleet. IECON 2014 - 40th Annual Conference of the IEEE Industrial Electronics Society (Oct. 2014), 3522-3528.

[6] Sassi, O. and Oulamara, A. 2017. Electric vehicle scheduling and optimal charging problem: complexity, exact and heuristic approaches. International Journal of Production Research. 55, 2 (Jan. 2017), 519-535. DOI:https://doi.org/10.1080/00207543.2016.1192695.

[7] Wang, R. et al. 2016. Two-Stage Mechanism for Massive Electric Vehicle Charging Involving Renewable Energy. IEEE Transactions on Vehicular

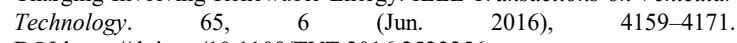
DOI:https://doi.org/10.1109/TVT.2016.2523256.

[8] Xi, X. and Sioshansi, R. 2014. Using Price-Based Signals to Control Plugin Electric Vehicle Fleet Charging. IEEE Transactions on Smart Grid. 5, 3 (May 2014), 1451-1464. DOI:https://doi.org/10.1109/TSG.2014.2301931. 